\title{
Double-double bend achromat cell upgrade at the Diamond Light Source: From design to commissioning
}

\author{
R. Bartolini, ${ }^{1,2}$ C. Abraham, ${ }^{1}$ M. Apollonio, ${ }^{1}$ C. P. Bailey,${ }^{1}$ M. P. Cox,${ }^{1}$ A. Day,,${ }^{1}$ R. T. Fielder, ${ }^{1}$ \\ N. P. Hammond, ${ }_{1}^{1}$ M. T. Heron, ${ }^{1}$ R. Holdsworth, ${ }^{1}$ J. Kay, ${ }_{1}^{1}$ I. P. S. Martin, ${ }^{1}$ S. Mhaskar, ${ }^{1}$ \\ A. Miller, ${ }^{1}$ T. Pulampong, ${ }^{1}$ G. Rehm, ${ }^{1}$ E. C. M. Rial, ${ }^{1}$ A. Rose, ${ }^{1}$ A. Shahveh, ${ }^{1}$ B. Singh, ${ }^{1}$ \\ A. Thomson, ${ }^{1}$ and R. P. Walker ${ }^{1}$ \\ ${ }^{1}$ Diamond Light Source, Oxfordshire, OX11 ODE, United Kingdom \\ ${ }^{2}$ John Adams Institute, University of Oxford, Oxford OX1 3RH, United Kingdom
}

(Received 29 November 2017; published 8 May 2018)

\begin{abstract}
Diamond has recently successfully commissioned a major change in the lattice consisting of the substitution of a standard double-bend achromat (DBA) cell with a modified four-bend achromat (4BA) cell called "double-double bend achromat" (DDBA). This work stems from the original studies initiated in 2012 towards a Diamond upgrade and provides the benefit of an additional straight section in the ring available for insertion devices. This paper reviews the DDBA design and layout, the implications for technical subsystems, the associated engineering challenges and the main results of the commissioning completed in April 2017.
\end{abstract}

DOI: 10.1103/PhysRevAccelBeams.21.050701

\section{INTRODUCTION}

In the framework of the studies for a possible upgrade of the Diamond storage ring to a lower emittance lattice, several cell structures have been carefully considered and compared in recent years [1,2]. In the course of these studies a novel cell design was proposed based on a modification of the four-bend achromat (4BA) cell which promises to deliver simultaneously a tenfold reduction in emittance with an additional straight section per cell, hence doubling of the capacity of the ring for insertion device (ID) beam lines. The design is based on the splitting of the traditional 4BA cell to insert an additional straight section in the middle of each arc. In this way, the basic cell resembles a pair of double-bend achromats (DBAs) and so it was dubbed "double-double bend achromat" (DDBA). As a sidework stemming from these studies, it was later proposed to consider the insertion of just one DDBA cell in the existing Diamond storage ring with the main purpose of adding a new straight section and therefore a new beam line to the ring even if the impact on the emittance is minimal [3]. The additional straight section is built to host an ID and feeds a new beam line dedicated to protein crystallography [4]. The electron optics is designed to accommodate a standard Diamond in-vacuum ID with a magnetic length of

Published by the American Physical Society under the terms of the Creative Commons Attribution 4.0 International license. Further distribution of this work must maintain attribution to the author(s) and the published article's title, journal citation, and DOI.
$2 \mathrm{~m}$ with $5 \mathrm{~mm}$ minimum operating gap. The possibility of using a cryogenic permanent magnet undulator (CPMU) is part of future exploitation plans for this straight section.

Inserting a radically different DDBA cell into a ring which operates with a high degree of availability and reliability for external users inevitably involved challenges, compromises and risk. As regards accelerator physics aspects, the Diamond lattice modified in this way has a severe break in the symmetry of the optics which goes against the commonly accepted criteria based on the optimization of the beam dynamics using perturbative approaches to the analysis of the betatron motion. This modification adds on top of three comparably minor breaks that were introduced in the optics in the past years, in particular two straight sections with two vertical mini-beta sections and horizontal focusing optics in the straight section I13 [5] and I09 [6]. As a result, the Diamond lattice is highly nonsymmetric and, in this sense, it is unique among purpose-built third generation light sources. There were technological challenges also. Achieving high strength quadrupoles and sextupoles with good field quality was not straightforward, nor were the dipoles with relatively high field gradient. Achieving the required vacuum pressures in the narrow gap vessels could not be achieved in the way initially envisaged and a compromise had to be made. Vacuum vessels had to be a mixture of stainless steel and copper, which complicated the fabrication and made it difficult to obtain the required mechanical tolerances. This paper presents the design strategy of the DDBA cell, the associated technological challenges, the assembly and installation and the results of the beam commissioning 
which was concluded successfully in December 2016, with first light in the beam line achieved in April 2017.

\section{CELL DESIGN}

The common trend in the design of the next generation of low emittance lattices for light sources is based on the concept of the multibend achromat (MBA) cell [7]. These cells are based, approximately, on (M-2) theoretical minimum emittance (TME)-like cells, flanked at each end by a matching cell. The outer dipole magnets are shorter in order to match the dispersion invariant and achieve the TME value. A popular and effective variation on this theme is the so-called hybrid MBA [8] in which the outer pairs of dipoles create two "dispersion bumps" used for efficient correction of chromaticity and nonlinear dynamics.

In view of the need at Diamond to also increase the provision of straight sections usable for IDs, we have considered the option of breaking the MBA cell in the middle to insert an additional straight section of usable length. In the case of Diamond the standard in-vacuum ID vessel is $2.486 \mathrm{~m}$ long and therefore requires no less than $3.4 \mathrm{~m}$ free length between adjacent quadrupole magnets. It is clear that longer straight sections are preferable since this would allow space for longer IDs, however, in the matching of the linear optics, the constraints on the length of the new midcell straight section was one of the most difficult to reach. The cell layout is reported in Fig. 1 with a comparison to the existing DBA cell.

The optics functions of the 24-cells DDBA ring are reported in Fig. 2. The optics design has nicely tailored the optics function and dispersion function at the center of the midstraight section so as to allow the insertion of an invacuum ID operated at a minimum gap of $5 \mathrm{~mm}$, similar to the other in-vacuum IDs at Diamond. Following the ESRF-EBS idea [8], a dispersion bump was introduced between the dipole pairs in order to create ideal positions for the chromatic sextupoles to correct the chromaticity. However, when a single DDBA cell is inserted into the existing DBA lattice, the DDBA cell optics has to be matched to the existing optics function of the nearby straight sections, so as to render the insertion as transparent as possible in terms of linear optics, i.e. maintaining the same photon beam properties in the adjacent

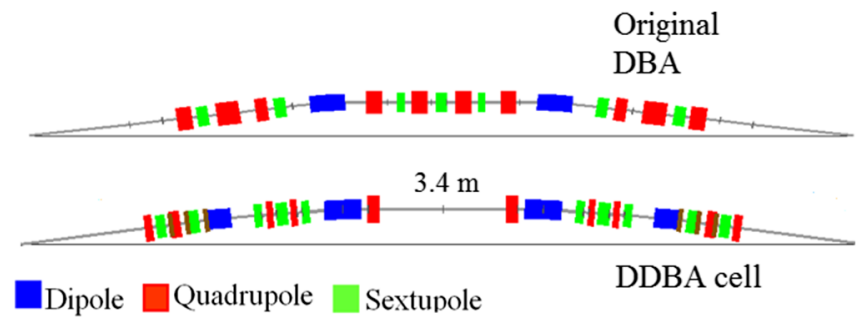

FIG. 1. DDBA layout compared to the existing DBA layout.



FIG. 2. Optics function of the DDBA cell for the complete ring upgrade of Diamond.

straight section. As a consequence, the dispersion function is not closed to zero in the adjacent straight sections, since the achromatic condition is already broken in all straight sections of the Diamond storage ring and the emittance of the ring is only slightly modified, with minimal perturbations to the rest of the ring. The cell is inserted between the existing flanges, thereby creating no space reduction on the existing adjacent straight sections. The value of the optics function in the adjacent straights is changed by $10 \%$ thereby creating a slight asymmetry in the adjacent straight sections. The table of the main parameters for the DBA lattice and the new lattice with the single DDBA modified cell are reported in Table I and the cell2 optics matched to the existing adjacent section is reported in Fig. 3. Due to the increased magnet gradients and lower beta functions within the DDBA cell, the tune point naturally increases by about 1 unit in the horizontal plane and 0.5 in the vertical when matched to the remainder of the ring. This vertical tune shift would naturally place the fractional tune above the half integer, which is unacceptable due to resistive-wall driven instabilities. As such, it was necessary to reoptimize the fractional part, and the final value was identified as providing a better working point. The circumference cannot be restored because we needed to maintain the alignment with the beam line in the additional midstraight section and the additional dipoles modified the reference orbit shortening the circumference.

TABLE. I. Comparison of main lattice parameters before and after the DDBA cell installation.

\begin{tabular}{lcc}
\hline \hline Parameter & Before DDBA & After DDBA \\
\hline Circumference $(\mathrm{m})$ & 561.600 & 561.571 \\
Harmonic number & 936 & 936 \\
Emittances $(\mathrm{H}$ and $\mathrm{V})$ & $2600 \mathrm{pm}-8 \mathrm{pm}$ & $2700 \mathrm{pm}-8 \mathrm{pm}$ \\
Betatron tunes & $27.21 / 13.36$ & $28.17 / 13.27$ \\
Natural chromaticities & $-79.4 /-38.1$ & $-78.8 /-41.2$ \\
\hline \hline
\end{tabular}




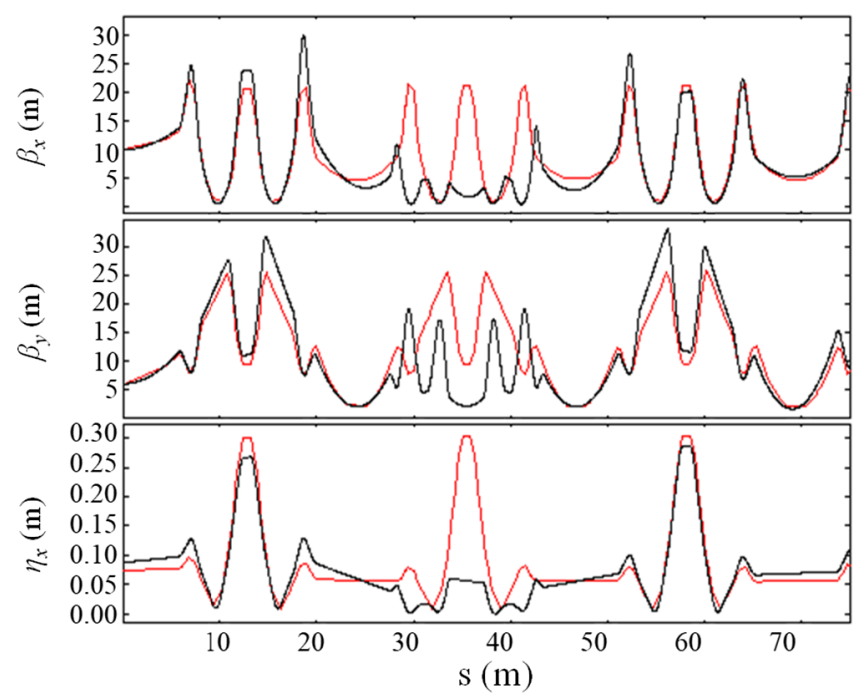

FIG. 3. Cell 2 optics matched to the existing adjacent cell: DBA optics (red) new optics (black).

The optimization of the nonlinear optics has been carried out using several different approaches. Due to the limited space and limited number of elements between the sextupoles in the DDBA cell, the prescriptions for the analytical compensation of the chromatic sextupoles (i.e. $3 \pi$ in horizontal phase advance and $\pi$ in the vertical) were not applicable. We therefore mainly resorted to a numerical optimization, using tracking calculations to estimate the dynamic aperture (DA), the momentum aperture (MA) and the frequency map (FM) with the aid of the most advanced numerical optimization tools based on multiobjective genetic algorithms. The direct calculation of the injection efficiency via tracking of the injected beam distribution from the booster was also used as an objective for the optimization. The tracking was mostly performed with the ELEGANT [9] tracking code. These techniques allow a very



FIG. 4. Existing (black) vs new dynamic aperture (cyan) calculated at the injection point (no errors).



FIG. 5. Existing momentum aperture (black) vs new momentum aperture (cyan).

accurate computation of the machine performance, including realistic alignment and field errors in the magnetic elements. As a result, it was possible to show that despite the symmetry breaking and the consequent loss of control over the first order resonant driving terms, it was still possible to identify a set of sextupoles that produce an acceptable DA and MA. Most of the simulations were performed taking directly into account the injection efficiency and the Touschek lifetime as objectives. The best solution shows a limited reduction of the dynamic aperture and a minor reduction in the momentum aperture as shown in Figs. 4 and 5. The momentum aperture shows a beating which is likely due to uncorrected chromatic functions. In fact the optimization of the off axis dynamics did not target such quantities but focused directly on the final lifetime

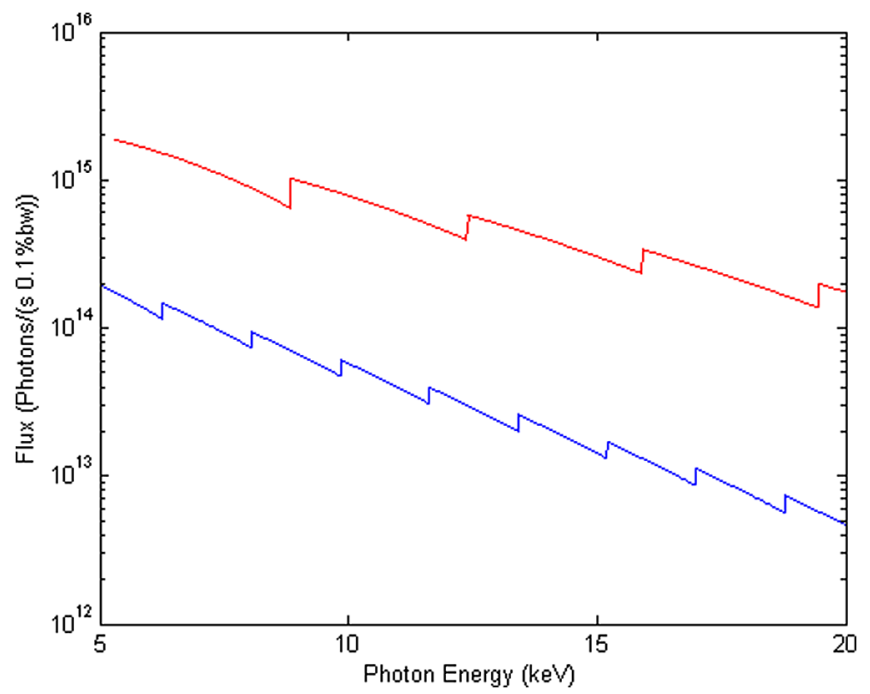

FIG. 6. Flux in the central cone for a short $0.7 \mathrm{~m}, 30.8 \mathrm{~mm}$ period, out-of-vacuum ID (blue), and a full length $2 \mathrm{~m} \mathrm{CPMU,}$ $17.7 \mathrm{~mm}$ period, in the DDBA straight section (red). 
and injection efficiency, which were found to be maximized if the strict, periodic constraints were relaxed. The effect on calculated lifetime and injection efficiency is acceptable, both reducing by approximately $15 \%$. These studies were performed assuming a set of random magnet misalignments and random field errors assigned to the new magnets in the cell which then informed the specification of the magnets.

The new midstraight section created by the DDBA design allows feeding the downstream beam line with a full length in-vacuum ID rather than a short out-of-vacuum ID (0.7 m long) as employed in another straight section in Diamond (I04). At present we have installed a $21 \mathrm{~mm}$ period in-vacuum ID, but the future plan is to install a CPMU [10]. Figure 6 reports the comparison in the flux between the originally proposed $0.7 \mathrm{~m} \mathrm{ID}$ and the planned CPMU showing clearly the larger flux from the CPMU, especially at photon energies as high as $20 \mathrm{keV}$.

\section{TECHNICAL SUBSYSTEMS}

We describe here the technical subsystems developed for the construction of the DDBA cell. It is worthwhile to note that the design originated from the study for a full upgrade of the Diamond storage ring to a 24-cell DDBA lattice. Hence, some subsystems, such as magnets and vacuum, served as prototypes for the technology that could be used for the full machine upgrade.

\section{A. Magnets}

As a consequence of the tight constraints on the optics functions and the limited space available, strong focusing magnets have to be introduced in the DDBA cell. In order to reduce the number of quadrupoles and create a compact layout, a vertical focusing gradient is introduced in the dipoles. The gradient dipoles have a dipole field of $0.8 \mathrm{~T}$ and a gradient of $14 \mathrm{~T} / \mathrm{m}$ and two dipoles length of 0.67 and $0.97 \mathrm{~m}$ are used. Standardization of the magnet design was considered in the early stages to simplify procurement and assembly and, as a result, the cell has ten quadrupoles with $70 \mathrm{~T} / \mathrm{m}$ maximum gradient, all $25 \mathrm{~cm}$ long, and ten sextupoles with $2000 \mathrm{~T} / \mathrm{m}^{2}$ maximum gradient, all $17.5 \mathrm{~cm}$ long. The sextupoles have embedded orbit correctors in both planes and skew quadrupoles for coupling correction. The space between the magnets is minimized wherever possible, while still reducing the cross talk between magnets and allowing sufficient space for further instrumentation, pumps and diagnostics. The minimum distance between elements was eventually set to $10 \mathrm{~cm}$ iron to iron.

The gradient dipole is based on a C-yoke with a pole designed with a Rogowski roll-off profile to reach the desired gradient with minimal saturation of the pole tip. The tight space constrains have led to the use of the socalled extended-pole concept [11] with an end design using nose overhangs on the poles and coils wound around the pole. Full 3D magnetic field simulations with OPERA [12] were carried out to design the pole face and ends to produce a good field region of $\pm 10 \mathrm{~mm}$ inside the aperture, consistent with the expected dynamic aperture. Beam dynamics simulations clearly highlighted that the sextupole term is the most detrimental for the beam dynamics and hence tight constraints were introduced. A chamfer was added at the pole end to control the relative strength of the most dangerous integrated multipolar components. The chamfer angle was optimized to reduce the sextupole term at the expenses of a slightly larger octupole term, which however was found to be less detrimental for the beam dynamics.

Each dipole has two sets of coils. Seven-turn coils are powered in series with the main dipole circuit, at a current of $1.4 \mathrm{kA}$ while the additional Ampere turns are provided by 12-turn trim coils from four individual $200 \mathrm{~A}$ power supplies. No additional trim coils were foreseen to decouple the value of the dipole field from the quadrupole field. As such, any eventual adjustment of their relative ratio requires the horizontal shift of the position of the dipole. The support structure was therefore designed to cater for a $\pm 2 \mathrm{~mm}$ overall horizontal shift. The 3D modeling of the gradient dipole revealed a slight difference between the magnetic length of the dipole and quadrupole components, resulting in a slightly weaker integrated quadrupole gradient. As a consequence, it was expected that to simultaneously set the correct dipole and quadrupole integrated values would require a slight horizontal offset of the magnet. More details on the alignment of the gradient dipoles are given in Sec IV.

The quadrupole magnets are designed to reach a gradient of $70 \mathrm{~T} / \mathrm{m}$. They have a small bore of $15 \mathrm{~mm}$ radius with a pole tip field of $1.05 \mathrm{~T}$. Pole chamfers $3 \mathrm{~mm}$ deep were used to control the systematic b6 (12-pole). The sextupoles should reach a maximum gradient of $2000 \mathrm{~T} / \mathrm{m}^{2}$, with a bore radius of $15 \mathrm{~mm}$. The pole tip field is $0.45 \mathrm{~T}$. Also in this case, $3 \mathrm{~mm}$ deep pole chamfers were used to control the systematic b9. Additional functionalities to provide $\mathrm{H}$ and $\mathrm{V}$ independent correctors and skew quadrupoles were added by winding additional coils around the poles in three independent circuits. The design of these small bore multipoles allowed a significantly reduced transverse envelope of these magnets. Figure 7 shows a comparison of the cross sections of the quadrupoles and sextupoles in the standard DBA cell with the new magnets in the DDBA cell.

All of the magnets were manufactured by Danfysik A/S. The gradient dipole and quadrupoles are built of solid iron, while the sextupoles are laminated to allow the operation of fast dipole correctors produced by additional corrector windings. To achieve the required high field quality, the pole tip profile of the quadrupole and sextupoles were machined with wire cut in a complete magnet block. The gradient dipoles were instead machined by milling. The coordinate measurement machine (CMM) measurements 



FIG. 7. Cross sections of quadrupoles and sextupoles in the existing DBA cell (right halves) and DDBA cell (left halves).

show a pole profile well within $20 \mu \mathrm{m}$ tolerance in the most sensitive area of the poles while $25 \mu \mathrm{m}$ tolerance was achieved for the gradient dipole poles.

The magnetic measurements, carried out during the factory acceptance tests, show some deviations with respect to the field quality predicted by the magnet simulations. However the errors are generally small, with unwanted harmonics limited to few units in $10^{-4}$ relative to the main field component at $\mathrm{r}=10 \mathrm{~mm}$, showing the overall good field quality of the magnets. Given the small amount of new magnets installed in the ring, it was possible eventually to analyze the impact of the magnetic errors on the beam dynamics using directly the measured values in the tracking simulations. The gradient dipoles were measured with a Hall probe, while the multipole harmonics were measured with a stretched wire [13]. The comparison between the measured harmonic and the design integrated harmonics is reported in Table II, which gives the ratio between the field component associated to the multipole of order $\mathrm{n}, \mathrm{b}_{n} \cdot \mathrm{x}^{n}$ and the main field component $\left(\mathrm{B}_{0}+\mathrm{gx}\right)$ computed at $\mathrm{x}=15 \mathrm{~mm}$. The grid used for the Hall probe measurement was too coarse to allow the precise measurement of the skew components.

Tables III and IV report the results of the stretched wire measurements in terms of the field harmonics at the

TABLE II. Magnetic measurement results for the DDBA gradient dipoles $(1.4 \mathrm{kA})$.

\begin{tabular}{lccc}
\hline \hline Component & $\begin{array}{c}\text { Mean } \\
\left(\times 10^{-4}\right)\end{array}$ & $\begin{array}{c}\text { Maximum } \\
\left(\times 10^{-4}\right)\end{array}$ & $\begin{array}{c}\text { Design values } \\
\left(\times 10^{-4}\right)\end{array}$ \\
\hline a3, b3 &,- 2.9 &,- 5.5 & $0,1.6$ \\
a4, b4 &,- 1.4 &,- 2.0 & $0,1.1$ \\
a5, b5 &,- 4.2 &,- 4.9 & $0,2.0$ \\
a6, b6 &,- 6.3 &,- 6.7 & $0,4.0$ \\
\hline \hline
\end{tabular}

reference radius of $\mathrm{r}=10 \mathrm{~mm}$ (scaled from measurements taken at $\mathrm{r}=13 \mathrm{~mm}$ ). The data are normalized to the main component, with $\mathrm{n}=1,2, \ldots$ referring to dipole, quadrupole and so on, while $\mathrm{a}_{n}$ and $\mathrm{b}_{n}$ refer to the skew and normal components respectively. The first three unallowed harmonics are reported with the first two allowed harmonics for each multipole type.

After two quadruple prototypes, all the errors were limited to the $10^{-4}$ range showing the good quality of the manufactured magnets. The sextupole 10-pole and 18-pole are larger than the specified maximum values but appear to be acceptable following further beam dynamics simulations.

TABLE III. Magnetic measurement results for the DDBA quadrupoles (170 A).

\begin{tabular}{lcccc}
\hline \hline Component & $\begin{array}{c}\text { Mean } \\
\left(\times 10^{-4}\right)\end{array}$ & $\begin{array}{c}\text { Rms } \\
\left(\times 10^{-4}\right)\end{array}$ & $\begin{array}{c}\text { Maximum } \\
\left(\times 10^{-4}\right)\end{array}$ & $\begin{array}{c}\text { Design } \\
\left(\times 10^{-4}\right)\end{array}$ \\
\hline a3, b3 & $0.1,0.3$ & $1.5,2.5$ & $4.0,3.7$ & 0,0 \\
a4, b4 & $0.0,0.4$ & $0.4,1.4$ & $1.2,3.2$ & 0,0 \\
a5, b5 & $0.1,-0.3$ & $0.2,0.5$ & $0.4,1.0$ & 0,0 \\
a6, b6 & $0.0,-0.3$ & $0.2,0.5$ & $0.4,1.3$ & $0,0.2$ \\
a10, b10 & $0.0,-0.3$ & $0.0,0.0$ & $0.1,0.4$ & $0,-0.2$ \\
\hline \hline
\end{tabular}

TABLE IV. Magnetic measurement results for the DDBA sextupoles (100 A).

\begin{tabular}{lcccc}
\hline \hline Component & $\begin{array}{c}\text { Mean } \\
\left(\times 10^{-4}\right)\end{array}$ & $\begin{array}{c}\text { Rms } \\
\left(\times 10^{-4}\right)\end{array}$ & $\begin{array}{c}\text { Maximum } \\
\left(\times 10^{-4}\right)\end{array}$ & $\begin{array}{c}\text { Design } \\
\left(\times 10^{-4}\right)\end{array}$ \\
\hline a4, b4 & $-0.1,1.3$ & $3.1,2.1$ & $5.0,4.7$ & 0,0 \\
a5, b5 & $-0.7,-3.5$ & $2.7,3.0$ & $5.2,8.8$ & 0,0 \\
a6, b6 & $0.0,0.6$ & $0.8,1.6$ & $1.6,4.6$ & 0,0 \\
a9, b9 & $0.1,-5.2$ & $0.2,0.5$ & $0.7,6.5$ & $0,-4.8$ \\
a15, b15 & $0,-1.7$ & $0,0.1$ & $0,-1.9$ & $0,-1.6$ \\
\hline \hline
\end{tabular}


The magnets were installed and aligned on two girders in a temperature stabilized assembly area outside the tunnel. The alignment of the gradient dipole is based on a set of mechanical fiducials given by three tooling holes, located on the top of the yoke, and two mechanical fiducials, located on a flat surface on the pole that can host magnetic cones. The relative location of the fiducials on the top of the yoke and on the pole were checked by measurements with a CMM. The latter were used to provide mechanical references to the grid with the magnetic field map, i.e. they allow transferring the grid built by the Hall probe bench to the magnet reference frame. The former are used to transfer the magnet reference frame to the girder reference frame: this is achieved by surveying in place the three fiducials on the top of the magnet yoke by means of a laser tracker. In this way, we can effectively survey the magnetic field map to the girder reference frame. The exact location of the magnetic field map is defined by computing numerically the trajectory of the electrons in the field map (with ACCELERATOR TOOLBOX [14]), making sure that the integrated field in the gradient dipole provides the nominal deflection angle and the nominal integrated quadrupole gradient. This process is done iteratively by adjusting the transverse offset of the dipole and recalculating the new trajectory until convergence is reached. Once this trajectory is known, it is transferred to the girder reference frame. This exercise provided the required magnet offsets needed to position the magnet in place on the girder. The integrated harmonics were calculated by expanding in Fourier series the integrated magnetic field computed on a circle in a plane orthogonal to the reference trajectory.

The multipoles were aligned on the girder using the same stretched wire system that had been used to validate the magnets at the manufacturer. The stretched wire system was used to find the magnetic center, pitch, roll and yaw of each magnet at the factory acceptance test, and to manufacture the shim sets that allow an accurate placement of the multipole magnets on the final girder, without a need for extensive remeasurement. The girder surface was designed to allow the installation of the stretched wire towers around the multipoles as shown in Fig. 8, and the system was used to confirm that the magnets were centered correctly in situ. This allowed errors in the shim sets to be corrected and ensured that each multipole was centered to \pm 25 microns with a roll of less than $0.1^{\circ}$.

The DDBA cell includes ten horizontal and vertical corrector magnets embedded in the sextupoles. Six of these correctors are located on stainless steel vessels while the remaining four are located over copper vessels that were introduced to manage the heat load of the radiation from the upstream dipoles. In order to sample the phase advance correctly, two additional discrete correctors are located in the new straight section thus providing adequate correction for the insertion device source position. Given the extremely tight space such correctors are only $10 \mathrm{~cm}$ long.

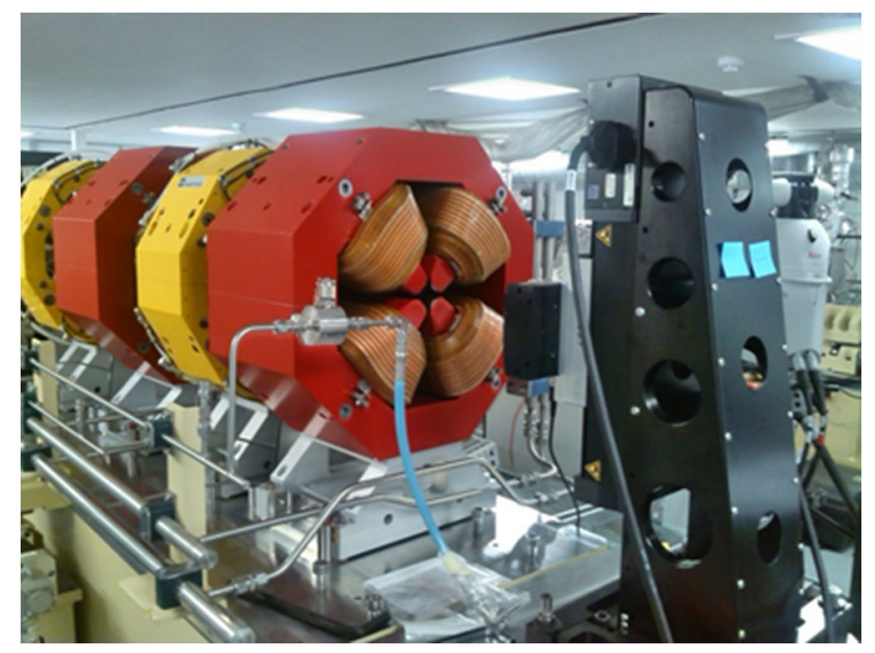

FIG. 8. Alignment of the magnets on the final girder with the stretched wire. One of the two towers of the stretched wire is visible on the right-hand side.

\section{B. Vacuum system}

The whole vacuum string in the DDBA cell had to be redesigned to take into account the new cell layout and the much reduced magnet apertures. In the design phase, the option of using a fully non-evaporable getter (NEG)-coated solution along the arc cell was considered in detail, but was deemed to require too much $R \& D$ given the size and timescales of the DDBA project. The main difficulties were identified to reside in the intricacies of uniform deposition and activation procedures for complicated vessel geometries, in the difficulties of providing a uniform heat distribution for NEG activation in such complex geometries, and in the relatively large number of different vessels involved. Furthermore, extensive vacuum pressure calculations showed that a vacuum system based on standard ion pumps and lumped NEG cartridges would be sufficient to guarantee the required vacuum performance, particularly in view of the fact that only a single cell was being modified in an already well-conditioned machine. As a consequence, we decided not to pursue a full-NEG-coating of the vacuum chamber but use discrete NEG cartridges and traditional lumped ion pumps at the crotches.

The final design of the vacuum pipe is made of four dipole vessels and several straight vessels along the beam path to cover the whole $17.35 \mathrm{~m}$ between the flanges in the arc. Two rf shielded gate valves are used in order to separate the newly created midstraight section. The vessels are made either of oxygen-free high thermal conductivity (OFHC) copper or 316L stainless steel: copper vessels are used downstream of the dipoles to take the synchrotron radiation heat load, while stainless steel vessels are used inside the dipole themselves and through some of the sextupoles that have embedded corrector magnets that form part of the fast orbit correction system.

The inner dimension of the vacuum chamber is a $27 \mathrm{~mm} \times 18 \mathrm{~mm}$ ellipse, much reduced compared to the 


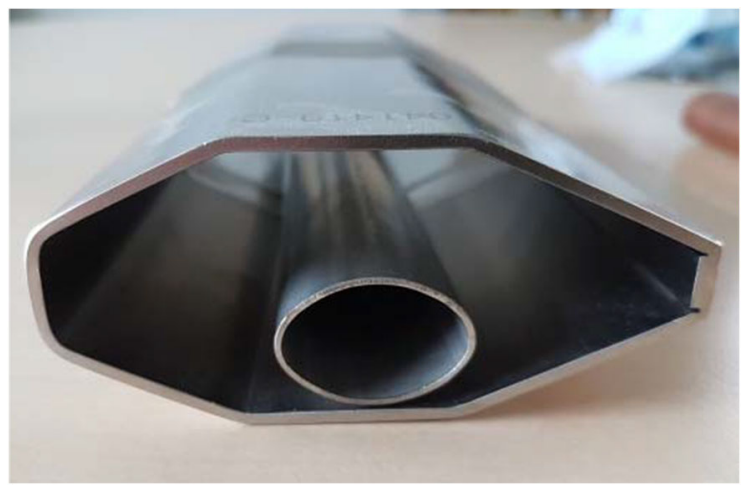

FIG. 9. Cross section of the DBA arc chamber (octagon) and DDBA arc chamber (ellipse).

$89 \mathrm{~mm} \times 38 \mathrm{~mm}$ octagonal chamber of the DBA cell (see Fig. 9). The chamber thickness is $1 \mathrm{~mm}$. The cross section is compatible with the reduced beam stay clear and provides $1.5 \mathrm{~mm}$ clearance of the chamber to the magnet pole tip to allowbakeout in situ. Water cooled distributed absorbers along the dipole vessel are used to absorb the synchrotron radiation and a number of wedges on one side of the inner surface of the pipe are placed in strategic positions to protect sensitive spots, like beam position monitors (BPMs) and flange joints from the upstream synchrotron radiation. This solution was considered preferable to having vessel antechambers given the tight space constraints in the assembly.

Different flange joint designs were examined. Driven by the requirement of reducing the flange gap as much as possible to avoid trapped modes and excessive rf heating, we explored the possibility of using spring-energized metal vacuum seals (Helicoflex $\left({ }^{\circ}\right.$ type) flanges rather than the Conflat $(\subset$ design, with knife-edge-type seal, which is used extensively at Diamond. In production, the surface finishing on the mating flanges in the seal region turned out to be critical and a number of iterations were needed before a satisfactory seal could be reliably obtained. It is still unclear whether such a solution can be reliably adopted on a larger scale project.

The pumping scheme consists of three sputter ion pumps at crotch absorbers downstream of dipoles 1, 3 and 4 and a series of seven NEG cartridges pumps. There was no space to fit a separate crotch absorber and ion pump downstream of dipole 2 and in that case the radiation is absorbed in the cooled wall of the elliptical chamber. A large $1500 \mathrm{l} / \mathrm{s}$ ion pump was installed nearby in the newly created straight section. Although not ideal and fully optimized, since the location of the pump is mostly dictated by the available space, nevertheless the final layout proved to be acceptable in terms of vacuum performance.

The vacuum design was driven by the requirement to achieve an average pressure of $10^{-9}$ mbar at an operating current of $300 \mathrm{~mA}$ after $100 \mathrm{Ah}$ conditioning dose. Vacuum simulations were performed with VACCALC [15], providing the 1D pressure profile along the cell. Published values were used for thermal outgassing rates and photon stimulated desorption yields [16]. The predictions show that the dynamic vacuum pressure distribution along the cell is contained to $10^{-9}$ mbar everywhere after 100 Ah of conditioning dose thus meeting the specification. It is worthwhile noting that the corresponding simulations with the fully NEG-coated chamber gave an average pressure of $10^{-9}$ mbar after only 9 Ah conditioning dose, showing the clear advantage of the fully NEG coated solution. While for one cell the solution proposed is acceptable, these studies point to a possible difficulty in extending this design for a full upgrade ( 24 cells) and a fully NEG coated solution might be preferable.

The vacuum chamber was baked out at the suppliers (FMB) at $200^{\circ} \mathrm{C}$ and then on the girders in the assembly area up to $180^{\circ} \mathrm{C}$, with heater jackets and kapton film heaters wrapped around the pipe, permanently fitted inside the magnet apertures. The static pressure was reduced easily to the $10^{-9} \mathrm{mbar}$ level, using a single $300 \mathrm{l} / \mathrm{s}$ turbomolecular pump. The vacuum performance with beam is described in Sec. IV below.

\section{Diagnostics}

The DDBA cell is equipped with a number of diagnostics to control beam position, track the physical location of the BPM blocks and beam losses, in line with the other cells. The specific features of the DDBA cell required the modification of the number and position of the correctors and BPMs for orbit correction and a complete redesign of the BPM block structure given the much reduced aperture of the vacuum pipe both in the arc and in the straight section.

Eight BPMs are used for orbit corrections instead of seven in the standard DBA cells. In total, six correctors over stainless steel vessels and two discrete short correctors over copper vessels are used in the fast orbit feedback [17]. The same BPM electronics is used in the new cell as in the existing cell, while the BPM's block and buttons had to be redesigned to fit the smaller cross section. The BPM buttons design is a compromise between producing lower signal power while at the same time better sensitivity to the beam offset and thus comparable performance. The $27 \mathrm{~mm} \times 18 \mathrm{~mm}$ aperture in the arc is incompatible with the existing BPM buttons diameter of $10.7 \mathrm{~mm}$ and new buttons with smaller diameter were investigated. Following the ESRF-EBS design, the button geometry has $6 \mathrm{~mm}$ diameter and $4 \mathrm{~mm}$ thickness with only one cylindrical gap between button and vessel. The cross sections of the existing and BPM buttons are shown in Fig. 10.

The design was aimed at reducing the impedance seen by the beam. Simulations with GDFIDL [18] show that the loss factors and trapped modes in the button structure were significantly reduced from $32.2 \mathrm{mV} / \mathrm{pC}$ for the current BPM block to $2.5 \mathrm{mV} / \mathrm{pC}$. Once in operation with beam, no significant heating was detected, confirming the 



FIG. 10. BPM block cross section Diamond DBA cell (left) and DDBA cell (right).

improved design, achieving a good compromise between sensitivity and wake losses.

All except two BPMs feature double or single bellows/ spring finger units to decouple them from any mechanical motion of the vacuum chamber as far as possible, and are equipped with optical position encoders which reference them to a nearby magnet or the floor.

\section{Engineering}

The original DBA cell is made of three girders supporting a total of two dipoles, ten quadrupoles, seven sextupoles, the vacuum pipe and instrumentation for vacuum and diagnostics, including seven beam position monitors and 16 ion pumps. The new DDBA cell is supported on two girders carrying four dipoles, ten quadrupoles, ten sextupoles, two additional short slow corrector magnets, eight BPMs, three ion pumps and seven NEG cartridge pumps.

The girders are $6.63 \mathrm{~m}$ long and they are built by Pro-Mil engineering with an overall flatness of $26 \mu \mathrm{m}$ over the whole length. A system of pin and dowel holes allows the alignment of the magnets on the girders with a nominal tolerance of $20 \mu \mathrm{m}$. The water manifolds, electrical connector panel and cable trays have all been incorporated at the design stage. Figure 11 shows a 3D model design of the fully preassembled girder and Fig. 12 their actual realization installed in the ring.

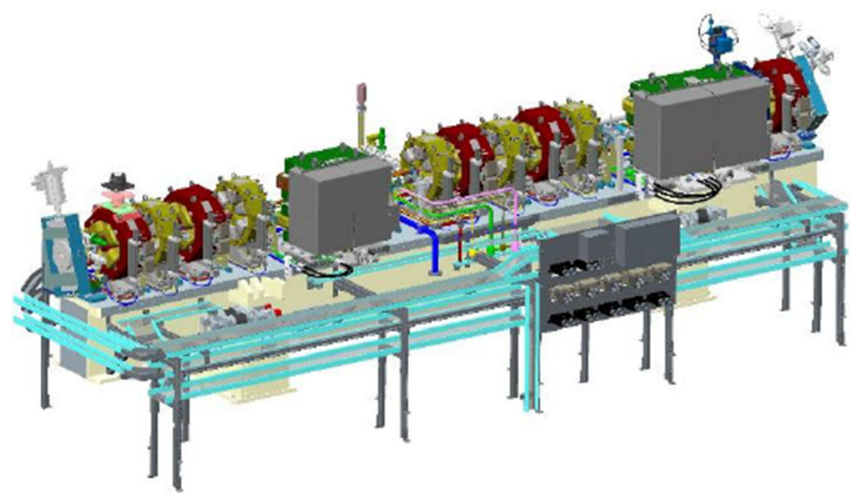

FIG. 11. 3D model of one of the DDBA girders [19].
Each girder sits on five cam movers which allow remote controlled adjustment for beam based girder alignment [20]. The concept is similar to the one used in the existing girders at Diamond, however, building upon the experience gathered at Diamond during the realignment program, the maximum travel generated by the cams was reduced to $\pm 1 \mathrm{~mm}$, to be within the maximum movement capacity of the bellows assembly and therefore prevent any damage. All of the magnet types were designed to be completely removable without the need to dismantle the vacuum vessels. This would allow replacement of any faulty coils which may occur during service without breaking the storage ring vacuum. Cooling water circuitry for the magnets and vessels was designed to minimize flow velocities and therefore reduce this source of vibration in the range $0.4-1 \mathrm{kHz}$.

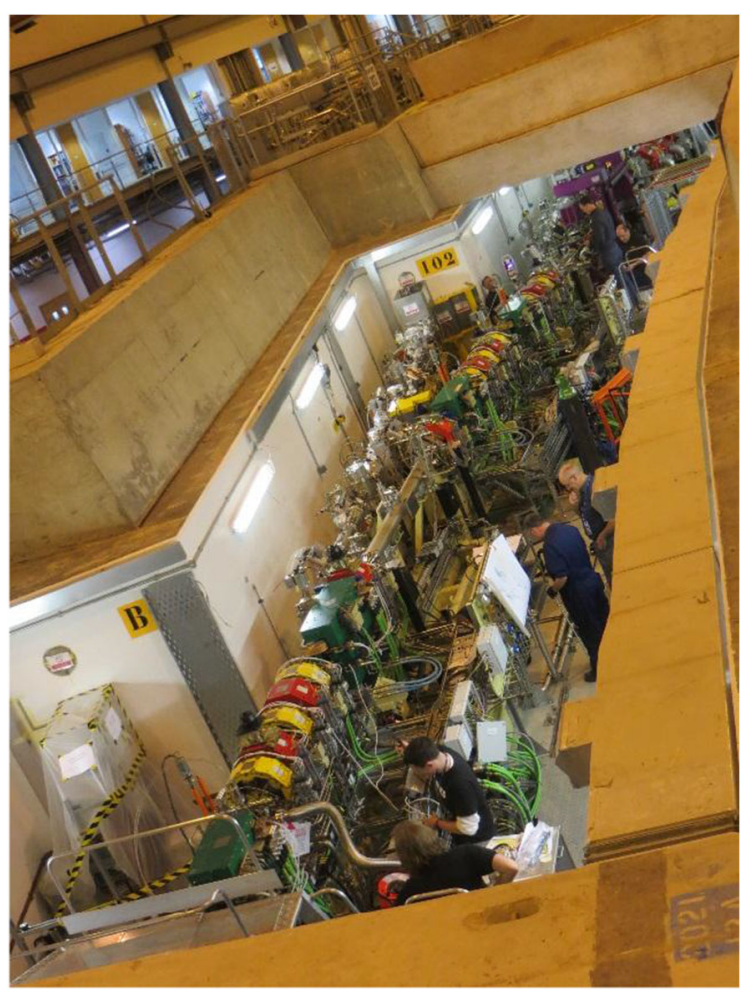

FIG. 12. DDBA girders installed in the tunnel. 
The girders were preassembled in a separate building which is $200 \mathrm{~m}$ from Diamond, including magnets, vacuum pipe, cabling and pipework, and were transported and installed in the tunnel fully assembled through the tunnel roof. The complete cell swap required the removal of the three girders of the standard DBA cell. The installation of the two new girders was planned to take place in eight weeks, by anticipating much of the cabling work in the previous six machine shutdowns [21]. It turned out that the disassembly procedure was faster than expected and the machine was ready for beam almost a full week in advance of the scheduled time. The girders were surveyed in place in the tunnel with a laser tracker.

\section{COMMISSIONING RESULTS}

The DDBA cell commissioning started with the assumption that no significant errors in the machine performance (e.g. orbit and optics) were expected to come from the rest of the machine, which was essentially unchanged from the previous operating period. The commissioning procedure was carefully prepared along the following steps [22]: (i) establishing first turn on axis; (ii) single shot on-axis capture, with rf on; (iii) establishing off-axis injection; (iv) accumulation; (v) orbit correction, beam based alignment; (vi) optics correction with Linear Optics from Closed Orbit (LOCO).

The whole procedure was underpinned by a thorough preparation and test of the high level software, based on MATLAB middle layer $[23,24]$. A change in the rf frequency to reflect the path length difference was implemented [25] and was within the operating range of the timing system, the injector and the related equipment (e.g. diagnostics, feedback systems, and so on).

Particular attention was given to the setting of the gradient dipoles. The strategy was based on starting the commissioning with the nominal current values in order to store the beam with the nominal quadrupole gradient. While a few turns were established relatively quickly, it was clear that the corrector magnets in the DDBA cell were too strongly excited, all with the same (negative) sign, pointing to an overall weakness in the dipole field. Raising the current in the dipole by $0.65 \%$ allowed the orbit to be fully corrected. The first attempt to correct the linear optic using LOCO, including the quadrupole gradient in the dipole as fit parameters, indicated that the gradients were still too low by $0.5 \%-1 \%$. After a number of iterations it was possible to bring the beta beat down from an initial $\pm 50 \%$ and $\pm 25 \%$ in the horizontal and vertical planes respectively, to below $5 \%$ in both planes, and the injection efficiency raised to $80 \%$. The correction to the magnet strengths was however insufficient to completely remove the negative bias of the horizontal corrector in the DDBA cell. As a consequence, we decided to enter the tunnel and move the gradient dipoles horizontally. A model based response matrix was used to calculate the best realignment
TABLE V. Adjustments made to the position of the dipole magnets and the gradient (magnet current) following beam based measurements.

\begin{tabular}{lcc}
\hline \hline Magnet & Displacement $(\mathrm{mm})$ & $\Delta \mathrm{K}(\%)$ \\
\hline Dipole 1 & +0.7 & +1.9 \\
Dipole 2 & +0.5 & +1.5 \\
Dipole 3 & +0.8 & +0.8 \\
Dipole 4 & 0 & +2.1 \\
\hline \hline
\end{tabular}

for each dipole keeping the gradients fixed. The procedure was done carefully by moving first only a single dipole and comparing the predicted change in the corrector pattern. The resulting agreement with the predicted reduction of the corrector strength gave us the confidence to move two more dipoles, while the last one was already close enough to its required position and so was not moved. Table $\mathrm{V}$ reports the final horizontal displacement of the gradient dipole and the gradient changes that were applied by following this beam based procedure. The reason why these changes were necessary in order to correct the linear optics is still under investigation. The corrector pattern before and after the dipole realignment is reported in Fig. 13.

Once stored beam was established, the accelerator physics studies concentrated on a sequence of orbit corrections, beam based alignment of BPMs to quadrupoles center and optics corrections with LOCO. At every cycle of orbit correction and beam based alignment the orbit correction visibly improved resulting eventually in a residual orbit below $1 \mu \mathrm{m}$ rms. Optics correction with LOCO showed a residual beta beating of $10 \%$ that was reduced to $2 \%$ after correction. Linear coupling was also successfully reduced. While initially it was found with LOCO that two consecutive skew quadrupoles tended to fight each other, it was decided to pair them and this reduced their relative excursion. Injection at $75 \%$ efficiency was routinely established with minor degradation in the performance as compared to before the DDBA installation which was routinely $85 \%-90 \%$.

Comparisons of the measured dynamic aperture before and after the DDBA installation are reported in Fig. 14 and confirm a slight degradation in the horizontal plane. While this is broadly in line with what was predicted by the model in Fig. 4, it is apparent that the model overestimates the boundary of the DA preliminary beam-based measurements indicate the final motion is limited by a $6 \mathrm{Q}_{x}$ resonance which is only weakly excited in the model. Efforts to understand this and to restore the horizontal dynamic aperture to the previous value are under way. We note, however, that the reduction in the boundary of the DA does not imply immediately the reduction of the injection efficiency, since the injected beam enters the machine at a nominal offset of $-6.8 \mathrm{~mm}$. The vertical dynamic aperture remains unchanged, and is limited by the physical apertures imposed by the ID straight sections and is therefore unrelated to the installation of the DDBA cell. Further 

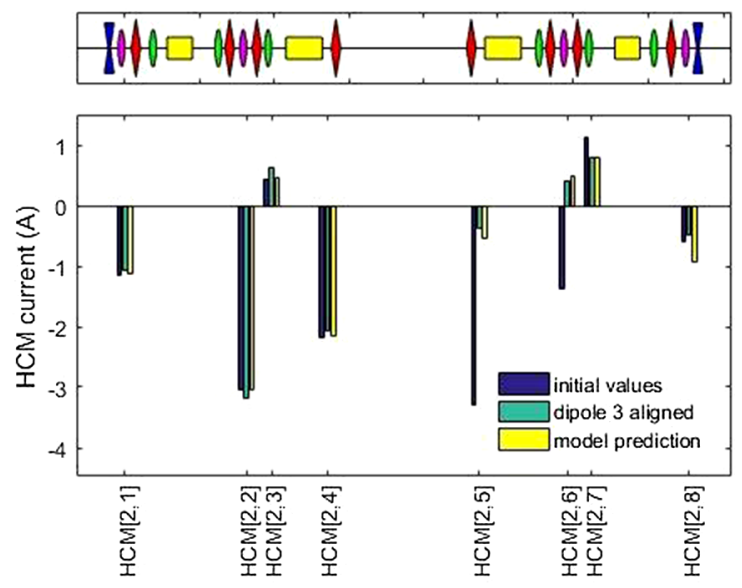
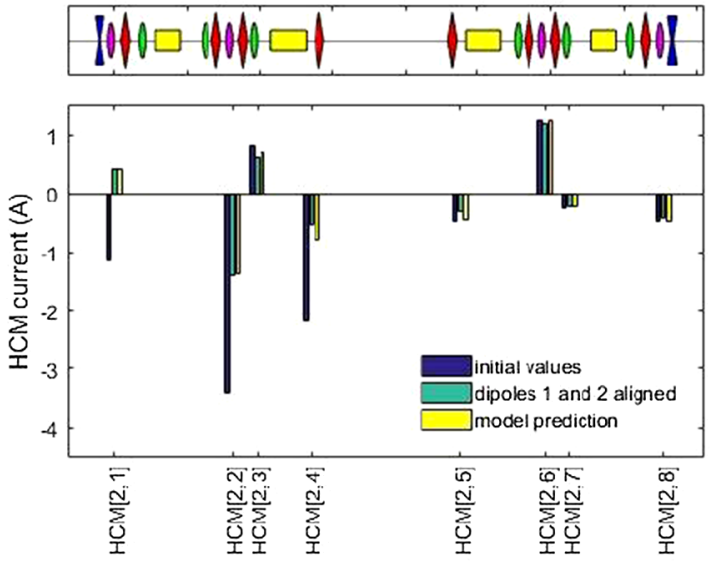

FIG. 13. Corrector pattern before and after the gradient dipole realignment.

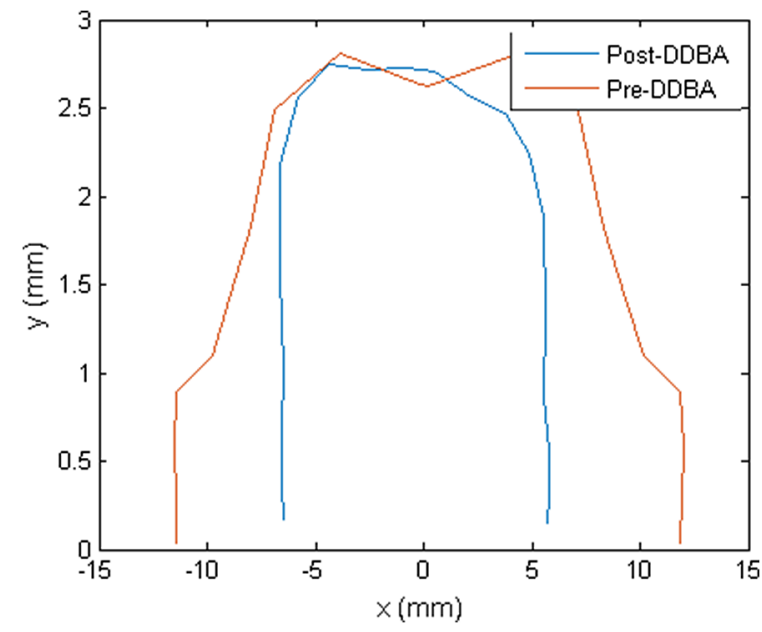

FIG. 14. Measured dynamic aperture before (red) and after (blue) the installation of DDBA cell, scaled at the injection point.

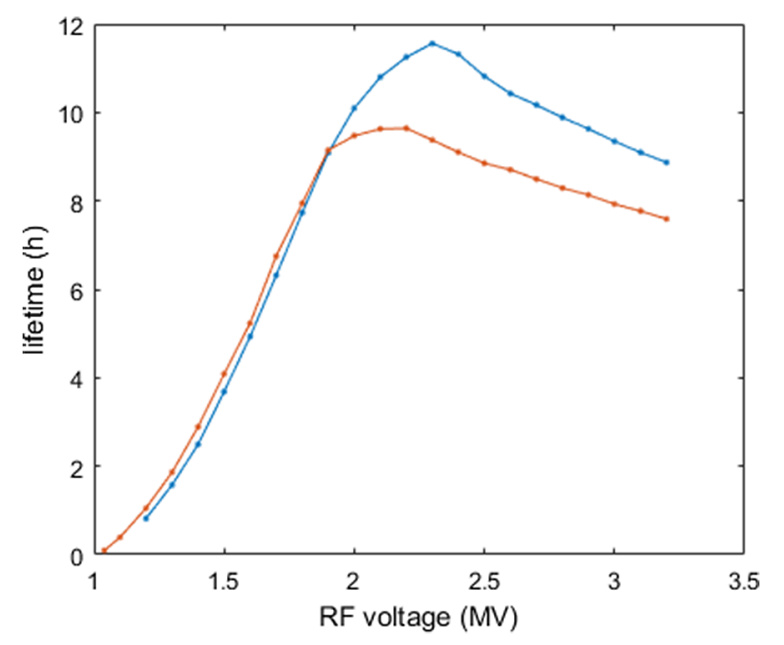

FIG. 15. Measured Touschek lifetime before (blue) and after the installation of DDBA cell (red). characterization of the machine performance was obtained by measuring the Touschek lifetime as a function of the rf voltage in operating conditions where the beam lifetime is dominated by Touschek scattering (10 mA, 100 bunches, low coupling at $0.1 \%$ ). The Touschek lifetime is reduced from a peak of 11.5 to $9.5 \mathrm{~h}$ as reported in Fig. 15 consistent with the predictions shown in Fig. 5. A slight shift in the curve towards lower voltages is also visible and results from the reduced energy loss per turn from the new cell. A closer comparison with the expected results from the model will be the subject of forthcoming investigations [26].

The whole commissioning procedure outlined here took only three days of normal shift operation. A few more days were spent in ramping up the current and after one week the machine was operating at the nominal $300 \mathrm{~mA}$. Vacuum and impedance related effects were carefully monitored during the process.

Vacuum conditioning proceeded well. Figure 16 shows the steady decrease of the dynamic vacuum pressure with the integrated beam current (beam dose). Correspondingly the beam loss rate normalized with current also decreased with dose up to $70 \mathrm{Ah}$, after which the lifetime become dominated by Touschek scattering.

The possibility of rf heating was watched closely by monitoring the temperature of critical components. It was found that flanges tended to increase in temperature but well within the operational limit and no serious concerns were raised. While these temperature observations did not point to any special issue coming from ray tracing and $\mathrm{rf}$ heating, a number of detailed measurements of impedance related effects show a general increase in the impedance content of the machine due to the narrower aperture vessel of the DDBA cell. A first analysis of the impact of collective instabilities was made by measuring the coupled-bunch instability thresholds with grow-damp experiment with a full multibunch fill before and after the DDBA installation. The growth rate of the multibunch modes is reported in Fig. 17, with a best fit model of the 


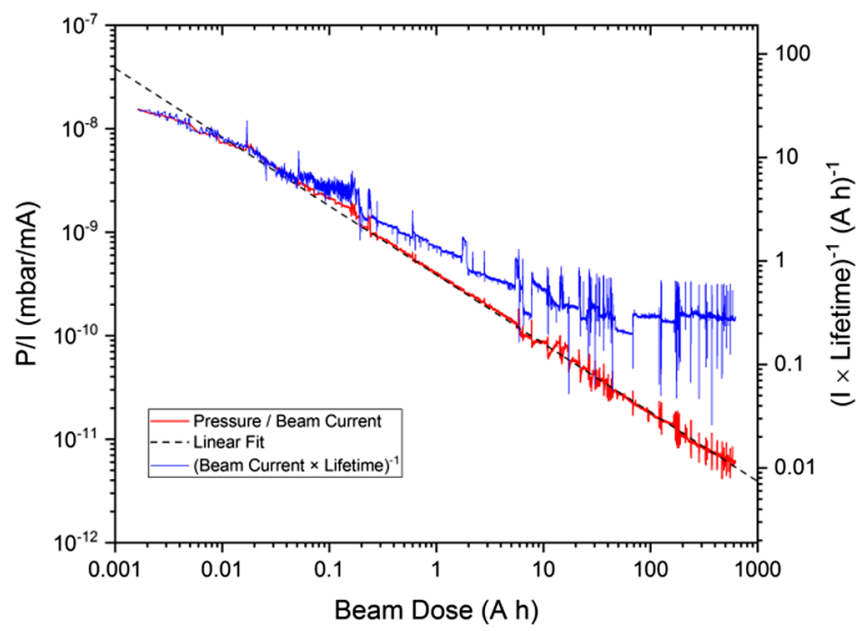

FIG. 16. Dynamic vacuum pressure averaged over the cell (red), $\mathrm{P} / \mathrm{I}$ where $\mathrm{P}$ is the pressure and $\mathrm{I}$ is the beam current; electron loss rate normalized to current (blue) following the DDBA installation [16].

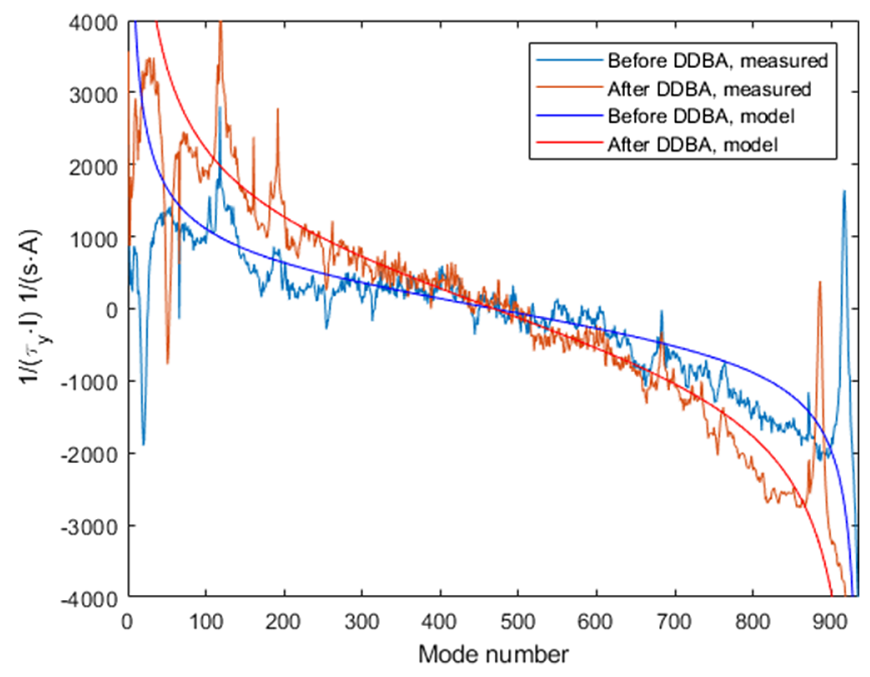

FIG. 17. Comparison of vertical multibunch modes growth rate before and after DDBA. All ID closed at $7 \mathrm{~mm}$ gap.

growth rate driven by the resistive wall impedance (solid lines). It is clear that there is an overall increase of the resistive wall contribution, while the resonatorlike structures are roughly unchanged. Quantitative comparison of these data will be the subject of forthcoming investigations, however from this first analysis it is clear that the impact of the single DDBA cell on the impedance is noticeable and it poses some question on the viability of this approach for a full lattice.

The installation of an ID in the newly created straight section was subsequently completed in the March 2017 shutdown and first light in the beam line was achieved in April. The beam line has been signed off, achieving the expected performance in terms of photon flux. The beam lines in the nearby straight sections registered a minor change in flux, again in line with what was predicted by the modification of the electron optics in the nearby sections. In particular the upstream beam line I02 suffered a reduction of about $10 \%$ in photon flux [27] due to a larger horizontal divergence of the electron beam at the source point.

\section{CONCLUSION}

The most extensive change to the Diamond storage ring since it became operational in 2007 has been successfully carried out. A whole DBA cell was successfully removed and replaced by a DDBA cell during a scheduled two-month shutdown from Oct. 7th to Dec. 5th of 2016. Standard user operation was resumed on Dec. 6th at $300 \mathrm{~mA}$ in top-up mode with no impact on reliability. The DBA cell provided an additional straight section in which an in-vacuum ID was installed in March 2017, giving "first light" to a new beam line in April 2017.

This modification has created a strong break in the symmetry of the lattice, contrary to all commonly held wisdom on symmetric lattice design, which makes the existing Diamond ring unique among purpose-built third generation light sources. As a by-product, the upgrade has also served as a technology test bed for a number of new solutions which are proposed for different subsystems in the next generation of low emittance rings, namely high gradient magnets, small aperture vacuum chambers, demanding assembly, installation and alignment procedures and so on. The lessons learned point to some open questions which might still offer potential risks for a full upgrade of the machine, most notably in the vacuum chamber design and the associated collective instabilities.

\section{ACKNOWLEDGMENTS}

We thank the many staff of Diamond Light Source who have contributed to the DDBA project and helped to make it a success. This project has received funding from the European Union's Horizon 2020 Research and Innovation program under Grant Agreement No. 730871.

[1] R. Bartolini et al., Novel lattice upgrade studies for diamond light source, in Proceedings of the 4th International Particle Accelerator Conference, IPAC2013, Shanghai, China, 2013 (JACoW, Shanghai, China, 2013), http://accelconf.web.cern.ch/AccelConf/IPAC2013/ papers/mopea068.pdf.

[2] R. Bartolini et al., Concepts for a low emittance-high capacity storage ring for the diamond light source, in Proceedings of IPAC2016, Busan, Korea, http://accelconf .web.cern.ch/AccelConf/ipac2016/papers/wepow045.pdf.

[3] R. P. Walker et al., The double-double bend achromat (ddba) lattice modification for the diamond storage ring, in Proceedings of IPAC2014, Dresden, Germany, http:// accelconf.web.cern.ch/AccelConf/IPAC2014/papers/mopro 103.pdf. 
[4] G. Evans and T. Sorensen, CDR for VMX (I02 \& I02.1), BLS-VMX-REP-002, 2012.

[5] B. Singh et al., Double mini-beta-y plus virtual focussing optics for diamond storage ring, in Proceedings of the 23rd Particle Accelerator Conference, Vancouver, Canada, 2009 (IEEE, Piscataway, NJ, 2009), http://accelconf.web .cern.ch/AccelConf/PAC2009/papers/th6pfp033.pdf.

[6] B. Singh et al., Implementation of double mini-beta optics at the diamond light source, in Proceedings of the $2 n d$ International Particle Accelerator Conference, San Sebastián, Spain (EPS-AG, Spain, 2011), http://accelconf .web.cern.ch/AccelConf/IPAC2011/papers/wepc042.pdf.

[7] D. Einfeld et al., Design of a diffraction limited light source (DIFL), in Proceedings of the Particle Accelerator Conference, Dallas, TX, 1995 (IEEE, New York, 1995), http://accelconf.web.cern.ch/AccelConf/p95/ARTICLES/ TPG/TPG08.PDF.

[8] P. Raimondi, Hybrid multi bend achromat: from SuperB to EBS, in Proceedings of IPAC2017, Copenhagen, Denmark, http://accelconf.web.cern.ch/AccelConf/ipac2017/papers/ thppa3.pdf.

[9] M. Borland, elegant, APS LS-287, 2001.

[10] Z. Patel et al., Insertion devices at diamond light source: a retrospective plus future developments, in Proceedings of IPAC2017, Copenhagen, Denmark, http://accelconf.web .cern.ch/AccelConf/ipac2017/papers/tupab116.pdf.

[11] R. Gupta and A. Jain, Special magnet designs and requirements for next generation light sources, in Proceedings of the 23rd Particle Accelerator Conference, Vancouver, Canada, 2009 (Ref. [5]), http://accelconf.web.cern.ch/AccelConf/ PAC2009/papers/tu1rai01.pdf.

[12] OPERA 18R2, Cobham Technical Services, Vector Fields Software, Kidlington, UK.

[13] G. Le Bec, J. Chavanne, and Ch. Penel, Stretched wire measurement of multipole accelerator magnets, Phys. Rev. ST Accel. Beams 15, 022401 (2012).

[14] A. Terebilo, Accelerator modeling with MATLAB accelerator toolbox , in Proceedings of the Particle Accelerator Conference, Chicago, IL, 2001 (IEEE, New York, 2001), http://accelconf.web.cern.ch/AccelConf/p01/PAPERS/ RPAH314.PDF.

[15] B. F. Macdonald, S. Bryan, H. S. Shiers, and M. P. Cox, Storage ring vacuum system pressure modelling at Diamond, Vacuum 84, 283 (2009).

[16] M. Cox et al., Vacuum system for the diamond light source DDBA upgrade, in Proceedings of IPAC2017,
Copenhagen, Denmark, http://accelconf.web.cern.ch/ AccelConf/ipac2017/papers/wepva136.pdf.

[17] S. Gayadeen et al., Performance of the fast orbit feedback system with the double-double bend achromat installed in diamond light source, in Proceedings of IPAC2017, Copenhagen, Denmark, http://accelconf.web.cern.ch/ AccelConf/ipac2017/papers/tupik113.pdf.

[18] W. Burns, GdfidL: A finite difference program with reduced memory and CPU usage, in Proceedings of the Particle Accelerator Conference, Vancouver, BC, Canada, 1997 (IEEE, New York, 1997), http://accelconf.web.cern .ch/accelconf/pac97/papers/pdf/9P118.PDF.

[19] R. P. Walker et al., Preparations for the double-double bend achromat installation in diamond light source, in Proceedings of IPAC2016, Busan, Korea, http://accelconf.web .cern.ch/AccelConf/ipac2016/papers/wepow048.pdf.

[20] M. Apollonio et al., Progress on the transparent realignment of the diamond storage ring, in Proceedings of IPAC2017, Copenhagen, Denmark, http://accelconf.web .cern.ch/AccelConf/ipac2017/papers/wepab091.pdf.

[21] A. Thomson et al., Optimisation of electrical and instrumentation design for the diamond light source DDBA upgrade, in Proceedings of IPAC2017, Copenhagen, Denmark, http://accelconf.web.cern.ch/AccelConf/ipac2017/ papers/mopab133.pdf.

[22] I. P. S. Martin et al., Electron beam commissioning of the DDBA modification to the diamond storage ring, in Proceedings of IPAC2017, Copenhagen, Denmark, http://accelconf.web.cern.ch/AccelConf/ipac2017/papers/ wepab095.pdf.

[23] W. Rogers et al., Control system developments for the diamond light source DDBA upgrade, in Proceedings of IPAC2017, Copenhagen, Denmark, http://accelconf.web .cern.ch/AccelConf/ipac2017/papers/tupik115.pdf.

[24] G. Portmann, An accelerator control middle layer using MATLAB, in Proceedings of the 21st Particle Accelerator Conference, Knoxville, TN, 2005 (IEEE, Piscataway, NJ, 2005), http://accelconf.web.cern.ch/accelconf/p05/papers/ fpat077.pdf.

[25] C. Christou, Tuning of the injector system to match possible lattice upgrades at diamond light source, in Proceedings of the 4th International Particle Accelerator Conference, IPAC-2013, Shanghai, China, 2013 (Ref. [1]), http://accelconf.web.cern.ch/AccelConf/IPAC2013/papers/ mopea069.pdf.

[26] I. Martin et al. (to be published).

[27] K. McAuley (private communication). 